\title{
Mixed-mode delamination in laminate plate with crack
}

\author{
Eva Kormanikova*, Kamila Kotrasova
}

Department of Structural Mechanics, Technical University of Košice, Civil Engineering Faculty, Vysokoškolská 4, 04200 Košice, Slovakia

*Corresponding author

DOI: $10.5185 /$ amp.2018/1894

www.vbripress.com/amp

\begin{abstract}
This paper deals with numerical modelling of laminate composite plate delamination that consists of unidirectional fiber reinforced layers. The delamination may be a fundamental problem in design of composite structures. The first-order shear laminate plate theory and fracture and contact mechanics were adopted for the methodology. The sublaminate modelling and delamination modelling are described by the help of finite element analysis. The displacements, reaction forces in springs and energy release rate along the lamination front are computed in modelling of interface. The numerical results are presented for problems with delamination of the mixed mode by using of $2 \mathrm{D}$ finite element analysis in the commercial ANSYS software. Copyright @ 2018 VBRI Press.
\end{abstract}

Keywords: Composite laminate plate, delamination, finite element method, modelling.

\section{Introduction}

Fast development of composites materials, composite parts of structures and composite structures has provided the incentive for solution one of the most severe defects in such structures - delamination. The delamination may be caused by manufacturing imperfections, cracks produced by fatigue or low velocity impact, the stress concentration near hinges and free edges, or due to high interlaminar stresses.

The delamination can grow at the edge of interlaminar defects in laminates loaded in compression, due to a global or local buckling of layers [1]. In addition, the delamination can occur as a failure mechanism in rehabilitated structures resulting from interfacial defects between the repaired composite laminate made of layers reinforced by fibers and the existing concrete material system [2].

A more efficient alternative of analysis for delamination is to use plate theory. The classical delamination models [3-5] consider the laminate as a composition of two plates in the cracked zone and of a unique plate in the undelaminated region. The classical delamination models [3-5] may provide an accurate estimate of the total energy release rate $G$. Unfortunately they are not able to accurately predict the individual energy release rates due to inaccurate description of the local peak crack tip strain state and to take into account of shear deformations. As a result, the classic plate-based delamination models were improved by various methods. Delamination was modelled by accepting sublaminates controlled by transverse shear deformable laminate theory to obtain a reasonable approximation to the mode separation solution [6].

Many recent works dealing with delamination are focused on the problem of correct modeling of delamination initiation and delamination growth. Iannucci [7] suggests the modeling technique of interface for explicit Finite Element Methods (FEM). The stress threshold for damage commencement together with the critical energy release rate for particular delamination mode is used in the technique based on fracture mechanics. The mathematical approaches based on variation methods and energy solution allow to develop numerical algorithms for the analysis of the crack propagation process at the interface using of Boundary Element Method (BEM) [8]. The modeling technique of interface was applied to a series of common delamination tests to demonstration the approach of superiority over standard stress-based failure criterion.

The work [9] aims to determine the properties of high density fiber reinforced polyethylene composites in two ways:

- experimental analysis

- finite element analysis

\section{Crack driving force}

In 1957 Irwin [10] adapted the Griffith's model, for a purpose of technical practice. This new parameter was obtained by dividing of potential energy by the new crack area $\mathrm{d} A=t \mathrm{~d} a$, thus obtained parameter represents the energy needed for an incremental crack contribution. 


$$
G(u, a)=-\frac{1}{t} \frac{\partial W_{e}(u, a)}{\partial a}
$$

where $a$ is length of crack, $t$ is thickness, $u$ is relative displacement.

The relation (1) represents the elastic energy release rate (ERR) of the unit area of new crack.

Considering, the parameter $G$ as a derivation of the energy, thus the parameter has a meaning of the force.

For $G$ is summarized the following notation

$$
\begin{aligned}
& G<G^{c} \Rightarrow \mathrm{d} a=0 \\
& G=G^{c} \Rightarrow \mathrm{d} a \geq 0 \quad G>G^{c} \Rightarrow \text { forbidden. }
\end{aligned}
$$

After the introduction of the function $f(G)$

$$
f(G)=G-G^{c} .
$$

It can be formulated loading and unloading condition in the form

$$
f(G) \leq 0, \mathrm{~d} a \geq 0, \mathrm{~d} a f(G)=0 .
$$

The process of the crack propagation driven by the ERR governs the flow of the crack propagation at the interface according to the relation

$$
G=\partial \zeta\left(\frac{\zeta}{2}\right) k \Delta u^{2}
$$

where the parameter $\zeta$ express the scalar damage variable.

\section{Numerical experiment - finite element analysis}

In the numerical experiment is assumed, that the laminate composite plate consists of two sublaminates with thickness $h_{1}, h_{2}$, both are assumed to the in-plane dimensions. Every sublaminate is created by the system of plate elements of first order shear deformable theory that are bonded by zero-thickness interfaces in the transverse direction. Every element of the plate represents one or several physical fiber reinforced plies with their arbitrarily oriented material axes. Adhesion between the plates inside each sublaminate is enforced by using restrictive equations implemented through Lagrangian multipliers. The finite plate elements of first order shear deformation theory are joined at the interfaces inside every sublaminate using restrictive equations or rigid links characterized by two nodes and three degrees of freedom in node.

Perfect adhesion is supposed in the undelaminated region. The sublaminates are free to deflect along of the delaminated region but cannot to penetrate to each other. The constitutive equation of the interface includes two stiffness parameters, $k_{z}, k_{x y}$, respectively, that we consider as the penalty parameters. The relationship between the traction vector $\sigma$ components and the corresponding components of relative interface displacement vector is given in $[\mathbf{1 1}, \mathbf{1 2}]$

$$
\left(\begin{array}{l}
\sigma_{z x} \\
\sigma_{z y} \\
\sigma_{z z}
\end{array}\right)=\left(\begin{array}{ccc}
k_{x y} & 0 & 0 \\
0 & k_{x y} & 0 \\
0 & 0 & k_{z}
\end{array}\right)\left(\begin{array}{c}
\Delta u \\
\Delta v \\
\Delta w
\end{array}\right) .
$$

Relative opening, sliding and tearing displacements are given as the difference between displacements at the interface, it means between the lower and upper sublaminate.

The problem of ERR computation may be solved locally by meaning of variables of interface, it means interlaminar stresses and relative variables. It is necessary to do the interconnection between the interface approach and fracture mechanics approach. The connection is determined in such way that the interface approach corresponds to the limit physical situation when the thickness of a thin adhesive layer tends to zero. By using the interface constitutive equation (6) to calculating of interlaminar stresses leads to the following overall ERR expression [13]

$$
G=\frac{1}{2} \lim _{k_{z}, k_{x y} \rightarrow \infty}\left[k_{z} \Delta w^{2}+k_{x y} \Delta u^{2}+k_{x y} \Delta v^{2}\right], \quad \Delta w \geq 0
$$

where $G$ is the local ERR function along the delamination front.

The delamination grows on the delamination front region, if the following condition (8) is satisfied

$$
\left(\frac{G_{I}}{G_{I}^{C}}\right)^{\alpha}+\left(\frac{G_{I I}}{G_{I I}^{C}}\right)^{\beta}+\left(\frac{G_{I I I}}{G_{I I I}^{C}}\right)^{\gamma} \geq 1
$$

The mixed mode fracture parameters $\alpha, \beta, \gamma$ are determined by fitting to experimental test results. $G_{I}^{C}, G_{I I}^{C}, G_{I I I}^{C}$ are the considered material properties, independent of their location along the delamination front. $G_{I}^{C}, G_{I I}^{C}, G_{I I I}^{C}$ are evaluated from experimental procedures. The eq. (7) is obtained from interface model and may be used with the finite element method for checking of crack propagation. The extent of the delamination area propagation may be established by releasing in which the expression (8) is first satisfied, leading to modification of the delamination front. The analysis of the delamination growth have to be computed iteratively.

The separate components of ERR are computed by using

$$
\begin{aligned}
& G_{I}(A)=\frac{1}{2} \frac{R_{A}^{z} \Delta w_{B-B^{\prime}}}{\Delta_{n} \Delta} \quad G_{I I}(A)=\frac{1}{2} \frac{R_{A}^{n} \Delta u_{n B-B^{\prime}}}{\Delta_{n} \Delta} \\
& G_{I I I}(A)=\frac{1}{2} \frac{R_{A}^{t} \Delta u_{t B-B^{\prime}}}{\Delta_{n} \Delta_{t}}
\end{aligned}
$$

where $R_{A}^{z}, R_{A}^{n}, R_{A}^{t}$ are the spring element reactions of connecting node $A$ in the thickness direction (z), normal $(n)$ and tangential $(t)$ to the delamination front.

The relative displacements $\Delta w_{B-B^{\prime}}, \Delta u_{n_{B-B^{\prime}}}, \Delta u_{t_{B-B^{\prime}}}$ in direction $z, n, t$ are located between the nodes $B$ and $B^{\prime}$ immediately ahead of the delamination front along 
its normal directions passing through $A . \Delta_{t}$ and $\Delta_{n}$ are the characteristic sizes of mesh in the directions $(n)$ and ( $t$ ) (Fig. 1).

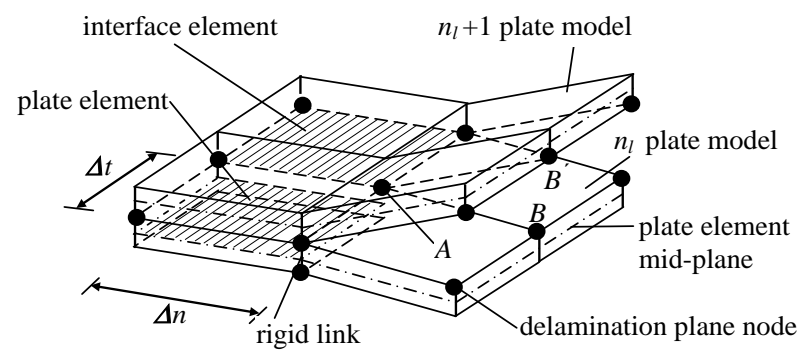

Fig. 1. Plate scheme in the neighborhood of the delamination front.

\section{Results and discussion}

The proposed model is verified for the square laminate plate consists of two sublaminates (1-1 model).

The subplates have different thicknesses. The upper subplate has $h_{1}=0.5 \mathrm{~mm}$, and lower subplate has $h_{2}=1 \mathrm{~mm}$. The subplates are affected by uniform load in each loading mode (Fig. 2). The plate geometry is seen from Fig. 3. The mechanical characteristics of the upper subplate (AS4D/9310) and the lower subplate (T300/5208) are shown in Table 1.

Table 1. The mechanical characteristics of the subplates.

\begin{tabular}{c|c|c}
\hline & $\begin{array}{c}\text { upper subplate } \\
(\mathrm{AS} 4 \mathrm{D} / 9310)\end{array}$ & $\begin{array}{c}\text { lower subplate } \\
(\mathrm{T} 300 / 5208)\end{array}$ \\
\hline$E_{x}[\mathrm{GPa}]$ & 134 & 136 \\
\hline$E_{y}[\mathrm{GPa}]$ & 7.7 & 9.8 \\
\hline$E_{z}[\mathrm{GPa}]$ & 7.7 & 9.8 \\
\hline$G_{y z}[\mathrm{GPa}]$ & 2.76 & 5.2 \\
\hline$G_{x y}[\mathrm{GPa}]$ & 4.3 & 4.7 \\
\hline$G_{x z}[\mathrm{GPa}]$ & 4.3 & 4.7 \\
\hline$v_{x y}$ & 0.3 & 0.28 \\
\hline$v_{x z}$ & 0.3 & 0.28 \\
\hline$v_{y z}$ & 0.4 & 0.15
\end{tabular}
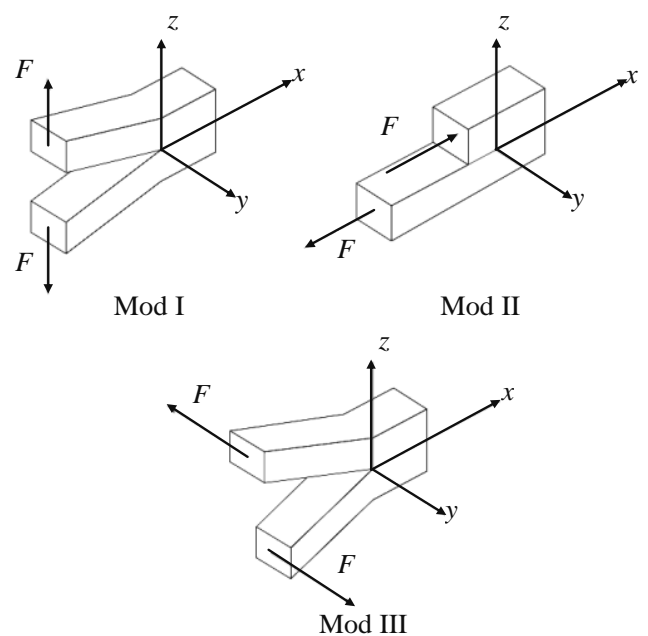

Fig. 2. Load modes I, II and III.

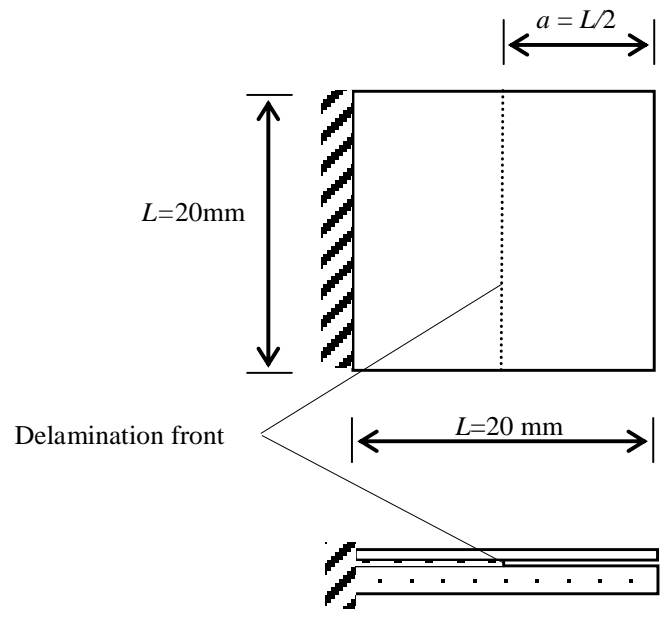

Fig. 3. Geometry of square plate.

The individual components of displacement vector for opening, shearing and tearing loading mode and response modes I, II and III for each load mode along the delamination front are plotted in Figs. 4, 5 and 6.

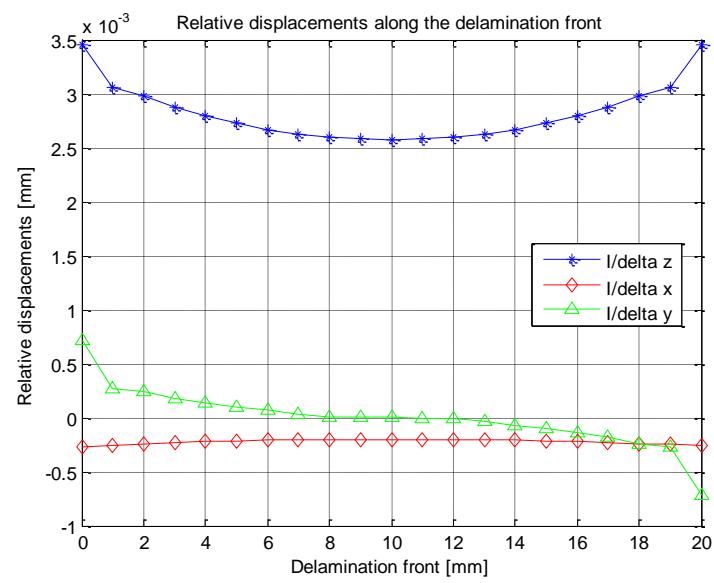

Fig. 4. Components of displacements through delamination front for load mode I.

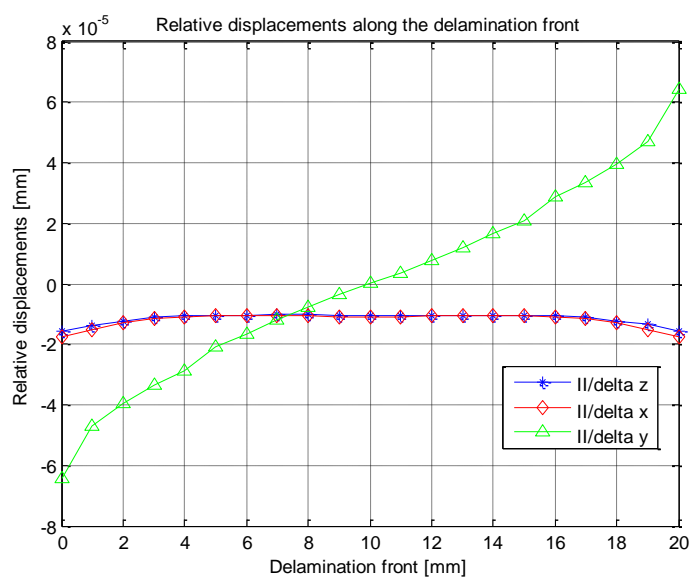

Fig. 5. Components of displacements through delamination front for load mode II. 


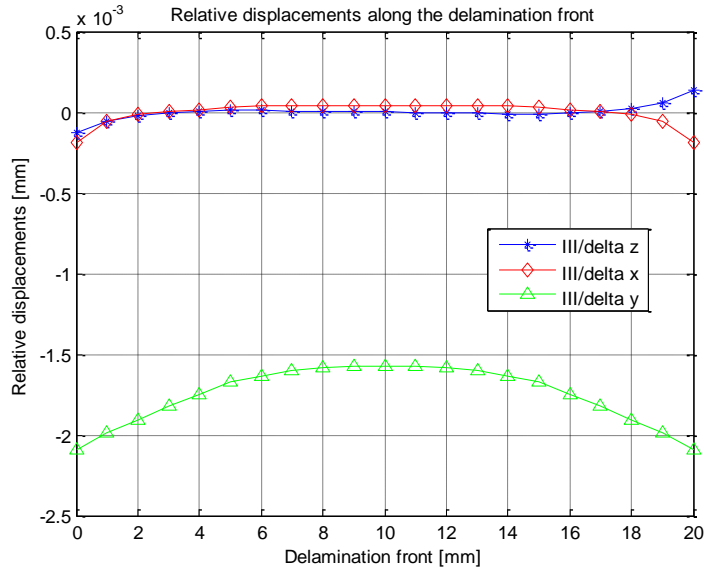

Fig. 6. Components of displacements through delamination front for load mode III.

The individual components of spring reaction forces for opening, shearing and tearing loading mode and response modes I, II and III for each load mode along the delamination front are plotted in Figs. 7, 8 and 9.

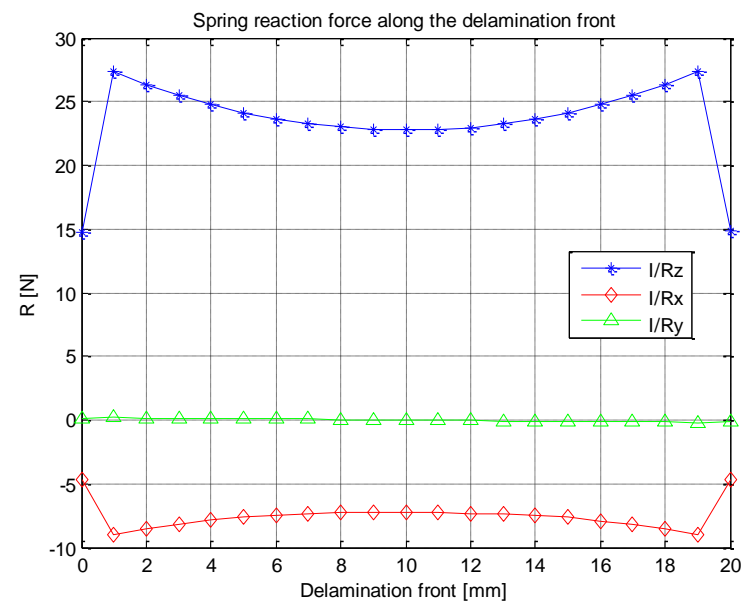

Fig. 7. Components of spring reaction forces through delamination front for load mode I.

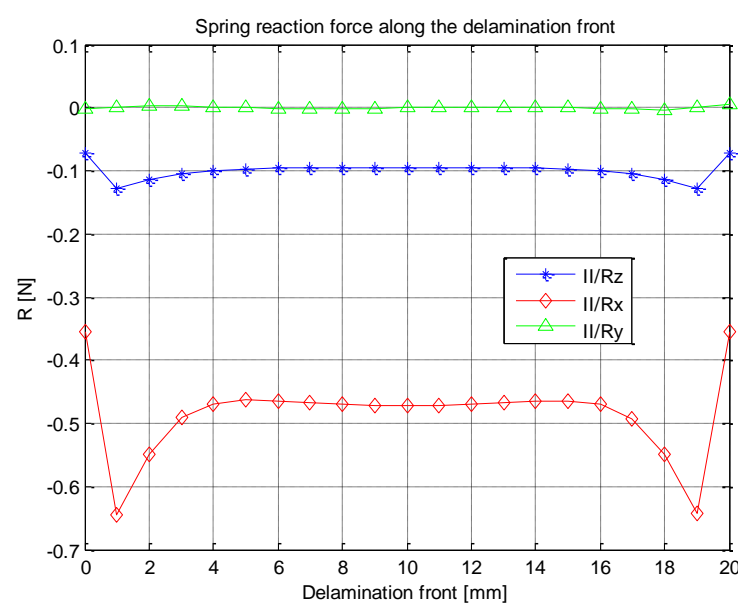

Fig. 8. Components of spring reaction forces through delamination front for load mode II.

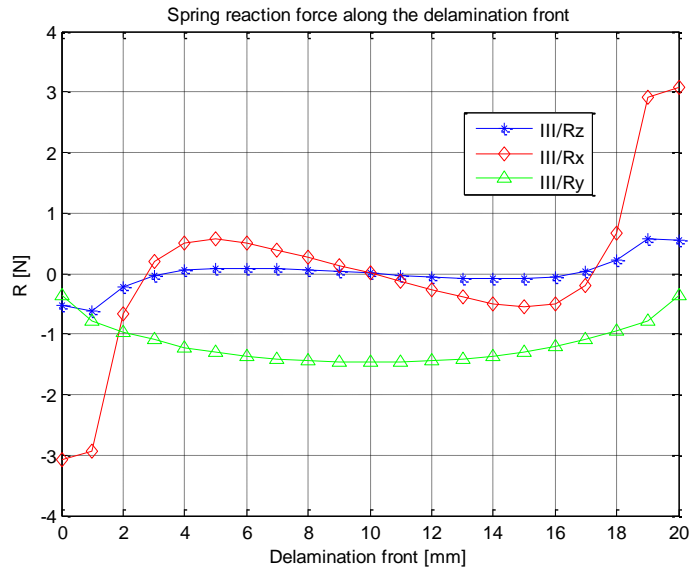

Fig. 9. Components of spring reaction forces through delamination front for load mode III.

The individual components of ERR for opening, shearing and tearing loading mode and response modes I, II and III for each load mode along the delamination front are plotted in Figs. 10, 11 and 12.

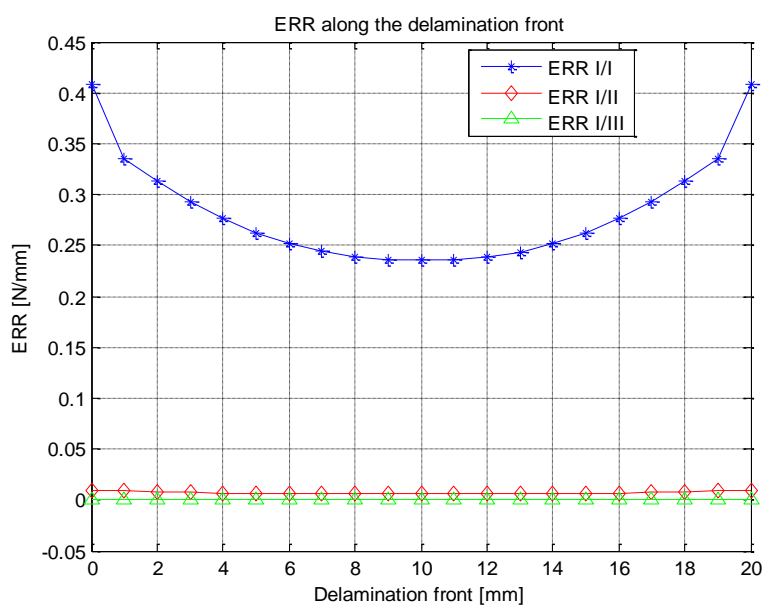

Fig. 10. Results of ERR components through delamination front for load mode I.

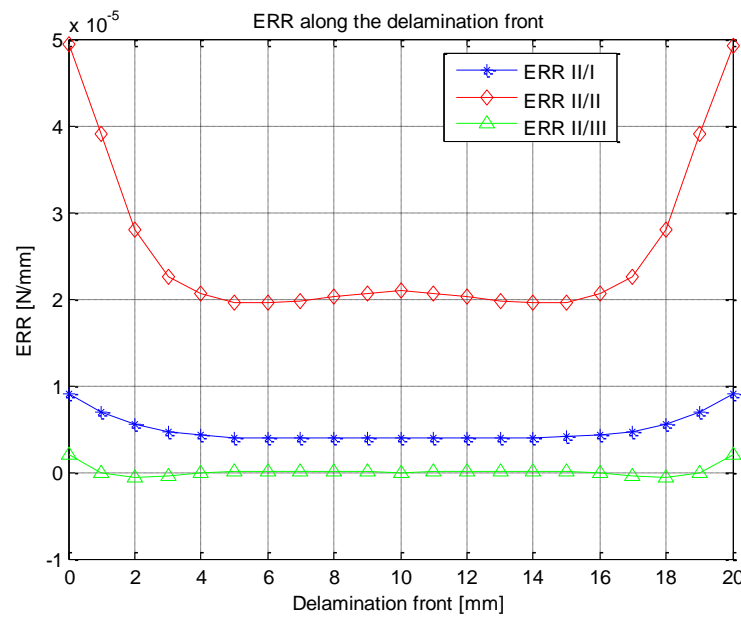

Fig. 11. Results of ERR components through delamination front for load mode II. 


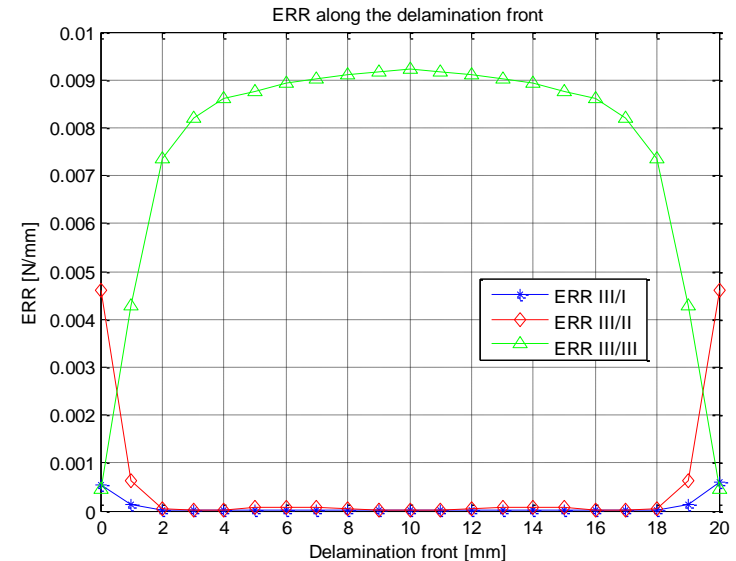

Fig. 12. Results of ERR components through delamination front for load mode III.

Two plates in the thickness direction were used to model the delamination of composite plate. The finite elements SHELL181 were used in the model. Projection on the plane of $x-y$ with finite element meshing, assumed to be the same for the plate and interface models of delamination front. The meshing zone of $5 \times 20 \mathrm{~mm}^{2}$ was centered with respect to the delamination front. The dimensions of plate and interface elements are $0.125 \mathrm{~mm}$ in the $x$-axis and $1 \mathrm{~mm}$ in the $y$-axis. In the $z$ (opening load mode), $x$ (sliding load mode) and $y$ (tearing load mode) direction, the force of $1 \mathrm{~N} / \mathrm{mm}$ is selected (Fig. 2). The interface elements required to connect the two subplates are realized by combining the two rigid links MPC 184, defined by two nodes with three degrees of freedom.

The front of the delamination was created by the COMBIN14 spring elements at each offset node of the front by three elements, and the offset nodes can be generated by rigid links. The interface was modeled by constrain equations $(\mathrm{CE})$. Using $\mathrm{CE}$ in place of rigid links, three coincident nodes located on the lower surface of the plate for each mid-plane node of the upper plate model, are created. Similarly, it also applies to the opposite way. Three elements (COMBIN14) acting in a differ direction of translation. Under the mixed mode response conditions, a complete modeling of the interface elements was performed. The stiffness of the spring elements that bind the subplates is equal to $10^{8} \mathrm{~N} / \mathrm{mm}^{3}$.

\section{Conclusion}

An orthotropic model contains of two subplates loaded by opening, sliding and tearing mode was proposed as failure model. This model has been modeled in program ANSYS by shear deformation theory. The response mode of displacements, spring reaction forces and energy release rate components along the delamination front were calculated.

The maximum values of results have been on the free edges of the model, so delamination is expected to occur from the free edges. From Figs. 10, 11 and 12 can be seen the following:
1. For opening load mode - the ERR I response mode is predominant and the modes II and III are negligible (Fig. 10).

2. For sliding load mode - the ERR II response mode is predominant and the mode III is negligible (Fig. 11).

3. For tearing load mode - the ERR III response mode is predominant and the modes I and II are negligible (Fig. 12) instead the free edges [14 - 19].

\section{Acknowledgements}

This work was supported by the Scientific Grant Agency of the Ministry of Education of Slovak Republic and the Slovak Academy of Sciences under Projects VEGA 1/0477/15 and 1/0078/16. The authors declare that they have no conflict of interest.

\section{References}

1. Bruno D.; Greco F.; Int. J. Solids Struct., 2001, 38, 9149. DOI: 10.1016/S0020-7683(01)00179-2

2. Lim Y. M.; Li V. C.; Cement Concr. Compos., 1997, 19, 373.

3. Cochelin B.; Potier-Ferry M.; Comput. Methods, Appl. Mech., $1991,89,361$.

DOI: $10.1016 / 0045-7825(91) 90048-\mathrm{B}$

4. Kim H. - J.; Comput. Structures, 1997, 62, 975 DOI: $10.1016 /$ S0045-7949(96)00290-8

5. Bruno D.; Greco F.; Int. J. Solids Struct., 2001, 38, 9149. DOI: $\underline{10.1016 / S 0020-7683(01) 00179-2}$

6. Zou Z.; Reid S. R.; Li S.; Journal of the Mechanics and Physics of Solids, 2001, 51, 333.

DOI: $10.1016 / \mathrm{S} 0022-5096(02) 00075-3$

7. Iannucci L.; Comput. Struct., 2006, 84, 1029

8. Kšiňan F.; Vodička R.; Kormaníková E; Proc. Eng., 2017, 190, 441.

9. Sivaraos, Leong, S.T.; Yusof, Y.; Tan, C.F.; Advances Materials Letters, 2015, 888.

DOI: $10.5185 /$ amlett.2015.5835

10. Irwin G. R.; Journal of Applied Mechanics, 1957, 24, 361.

11. Zmindak M.; Dekys V.; Novak P.; Adv. Mat. Res., 2014, 969, 176.

DOI: $10.4028 /$ www.scientific.net/AMR.969.176

12. Barbero E. J.; Finite Element Analysis of Composite Materials; CRC Press, Boca Raton, 2008.

ISBN-10: 1-4200-5434-1

13. Barbero E. J.; Introduction to Composite Materials Design; Taylor and Francis, NY., 1999.

ISBN: 15603270149781560327011

14. Michalcova V.; Lausova L.; Civil-Comp Proceedings, 2015, 108.

15. Tiwari A; Kobayashi, H. (Eds.); Responsive Materials and Methods; Wiley: USA, 2013. DOI: $10.5185 / \mathrm{sbm}-2010-01$

16. Krejsa M.; Koubova L.; Flodr J.; Protivinsky J.; Nguyen Q. T.; Frattura ed Integrita Strutturale, 2017, 11, 143. DOI: $10.3221 /$ IGF-ESIS.39.15

17. Mihalikova M.; Nemet M.; Vojtko M.; Key Eng. Mat., 2015, $635,118$. DOI: $10.4028 /$ www.scientific.net/KEM.635.118

18. Melcer J.; Lajcakova G.; Adv. Mat. Res., 2014, 969, 85. DOI: $10.4028 /$ www.scientific.net/AMR.969.85

19. Kormanikova E.; Kotrasova K.; Key Eng. Mat., 2015, 635, 85. DOI: $10.4028 / w w w . s c i e n t i f i c . n e t / K E M .635 .85$ 